\title{
Prevalencia y percepción del riesgo del tabaquismo en el área de salud del policlínico "Dr. Jorge Ruiz Ramírez"
}

\author{
Prevalence and perception of the smoking risk in the health \\ area cared for by "Dr Jorge Ruíz Ramírez" polyclinics
}

\author{
Nery Suárez Lugo'; Elizabeth Campos Pupo ${ }^{\text {II }}$
}

IDoctor en Ciencias de la Salud. Profesor Consultante y Titular. Escuela Nacional de Salud Pública. La Habana, Cuba.

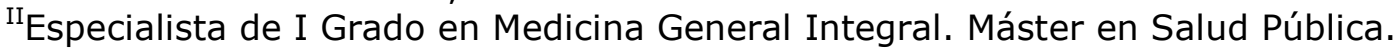
Policlínico Universitario "Dr. Jorge Ruiz Ramírez". La Habana, Cuba.

\section{RESUMEN}

Introducción El área de salud del policlínico "Dr. Jorge Ruiz Ramírez" presenta entre sus principales problemas de morbilidad y mortalidad las enfermedades que tienen al tabaquismo como factor de riesgo.

Objetivos Caracterizar la prevalencia, percepción del riesgo y las causas que inician y mantienen la adicción en la población de 10 años y más del citado policlínico.

Métodos Estudio descriptivo transversal realizado entre enero y diciembre del 2007. Se utilizó un muestreo por conglomerado bi-etápico y se empleó la encuesta como técnica para la obtención del dato primario.

Resultados Se obtuvo una prevalencia de tabaquismo de 38,2 \%, que fue mayor en el grupo de edad de 40 a 49 años, en el sexo masculino, en los individuos con nivel escolar universitario y en los trabajadores. Los fumadores se iniciaron en su mayoría en la adicción antes de los 20 años de edad por la influencia de amigos; la causa más mencionada para mantener la adicción fue el placer de fumar. El 93,9\% percibió que el tabaquismo es un riesgo, aunque demostraron desconocimiento de las enfermedades relacionadas.

Conclusiones. La prevalencia del tabaquismo en el área de salud del policlínico "Dr. Jorge Ruiz Ramírez", de acuerdo a la clasificación de la OMS, es elevada. El 
hábito se inicia en edades tempranas y se mantiene fundamentalmente por placer. Conocer esta situación así como la percepción de riesgo en la población estudiada, permite a las autoridades sanitarias disponer de información para trazar una adecuada estrategia de prevención y control.

Palabras clave: Tabaquismo, prevalencia, percepción de riesgo, Cuba.

\section{ABSTRACT}

Introduction The main morbidity and mortality problems existing in the health area cared for by "Dr Jorge Ruiz Ramírez" polyclinics are the diseases associated to smoking as a risk factor.

Objectives To characterize the risk prevalence and perception and the causes that encourage and keep smoking as addiction in the 10 years-old and over population cared for by this polyclinics.

Methods Cross-sectional descriptive study carried out from January to December 2007. Two-phase cluster sampling and survey as a primary data collection technique were used.

Results Smoking prevalence amounted to 38,2\%, being higher in 40-49 years-old group, males, individuals with university degree and simple workers. Most of the subjects started smoking before the age of 20 due to the influence exerted by friends; the most repeated cause of keeping the addiction was that they liked smoking. Among these subjects, 93,9\% admitted that smoking was a risk, but they did not know the diseases associated to this habit.

Conclusions Smoking prevalence is high in the health area cared for by "Dr Jorge Ruíz Ramírez" polyclinics according to the WHO classification. The habit started at early ages and is mainly kept because this population like smoking. Being aware of this situation and of the risk perception in the study population will allow the health authorities to have suitable information to outline suitable prevention and control strategy.

Key words: Smoking, prevalence, risk perception, Cuba.

\section{INTRODUCCIÓN}

El consumo crónico del tabaco constituye en la actualidad un fenómeno social que afecta indistintamente a todos los sectores de la población y constituye uno de los mayores problemas con que las autoridades sanitarias se enfrentan. Se considera una epidemia de carácter universal y es una gravosa carga para el individuo, la familia y la sociedad. ${ }^{1}$

El tabaquismo, definido como la intoxicación crónica producida por el abuso del tabaco, constituye la primera causa de mortalidad evitable, uno de los más importantes problemas que ponen en riesgo no solo la salud del individuo fumador, 
sino también la de las personas que conviven cotidianamente con fumadores, denominados fumadores pasivos.

La Organización Mundial de la Salud (OMS), en la última edición de la Clasificación Internacional de Enfermedades, ha catalogado al tabaquismo como una enfermedad crónica no trasmisible y adictiva. De esta forma, el consumo de tabaco se relaciona con más de 25 enfermedades de distintos síntomas y se estima que la asociación causal entre el tabaquismo y la mortalidad anual es de $30 \%$ con el cáncer general, así como con el cáncer nasal, oral, laringe, faringe, esófago, pulmón, mama, estómago, hígado, páncreas, riñón, uréter, vejiga, con las enfermedades cardiovasculares $25 \%$ y $18 \%$ con las cerebrovasculares. ${ }^{2-4}$

Las estadísticas de diversos países revelan que el consumo del tabaco comenzó a aumentar sostenidamente en los inicios del siglo $\mathrm{xx}$ y se intensificó desde la Primera Guerra Mundial. A pesar de que un reducido número de países mostraron un descenso de la prevalencia de fumadores durante las últimas dos décadas, lamentablemente la proporción de adolescentes y adultos jóvenes inician el consumo en edades entre 12 y 14 años. ${ }^{5}$

La OMS acordó en su reunión de 1986 que el tabaquismo y cualquier otra forma de uso del tabaco son incompatibles con el objetivo de "Salud para todos en el año 2000". Incluso, algunos expertos de la referida organización afirman que la lucha antitabáquica podría ser más útil para mejorar la salud y la prolongación de la vida, que el desarrollo de cualquier otro programa de actividades de medicina preventiva. ${ }^{6}$

Por las razones anteriormente expuestas, numerosos países se encuentran enfrascados en una lucha contra el tabaco, pues como problema global, se requieren estrategias globales para su solución. ${ }^{7}$ El Convenio Marco de la OMS para el Control del Tabaco (CMCT), adquirió fuerza jurídica obligatoria el 27 de febrero de 2005 y tiene como objetivo reducir el consumo en los jóvenes, contribuir a que los fumadores abandonen la adicción y proteger a los no fumadores de la exposición al humo ambiental. ${ }^{8}$

Aunque se ha avanzado en la educación sobre la nocividad del tabaquismo y su relación con la enfermedad y la muerte, estudios realizados en Cuba encuentran que los fumadores tienen conocimiento sobre el tabaquismo pero no percepción de sus riesgos. Esto corrobora que la información no resulta suficiente para cambiar conductas de salud, más aun cuando se trata de una droga que empleada de forma repetida, crea necesidad y hábito. ${ }^{9}$

La percepción de riesgo proporciona la capacidad de asumir gestiones protectoras en el individuo ante el tabaquismo, de ahí la importancia de disponer de información al respecto que permita realizar intervenciones efectivas a favor de la salud del individuo. En la actualidad, muchas investigaciones dirigen su atención hacia el conocimiento a profundidad del aspecto social de los riesgos, donde su percepción ocupa un destacado lugar.

Ejemplo de la falta de percepción del riego que conlleva el hábito de fumar, es el hecho de que el tabaquismo prevalece en los profesionales de la salud, que si bien han aprendido a reconocer el riesgo de eventos no saludables en sus pacientes, no siempre reconocen su propio riesgo de enfermar o morir. ${ }^{10}$

Es posible que las exigencias que plantea el medio sobrepasen los recursos actuales del sujeto y para él se convierta en una amenaza. Es de valor conocer que las actitudes influyen directamente en la percepción de riesgos. ${ }^{11}$ 
Es importante saber además, que la percepción de riesgo puede estar influenciada por elementos como la cultura, el género y experiencia anterior de la persona, ya que al igual que sucede con la salud física, las percepciones son el resultado de toda una vida de experiencias y desarrollo. El concepto de riesgo es eminentemente social y el individuo asume los riesgos de la población donde vive.

Diversos autores estiman que la percepción de riesgo y su aceptación, se rigen por ciertos principios como son:

- aún cuando existe un hecho concreto, muchas personas rechazan la presencia de una amenaza,

- en caso de ocurrencia de un desastre, las personas no cree que puedan ser dañados ellos o su familia en el mismo,

- creen en "algo" que les dará protección o los salvará,

- se tiende a pensar por muchas personas, que los beneficios serán mayores que los riesgos.

En el caso del tabaquismo, además de los riesgos conocidos, figuran otros derivados de las creencias equivocadas de los fumadores, pues se ha encontrado que unos consideran el tabaquismo como riesgo menor o despreciable, en algunos casos inclusive referente a que el cáncer de pulmón es igual o ligeramente mayor en los fumadores comparados con los no fumadores, tampoco consideran que el tabaquismo sea más mortal que los accidentes automovilísticos y creen que los cigarrillos con bajo contenido de alquitrán reducen los riesgos de cualquier enfermedad asociada al tabaco, y pasan por alto que fumar más cigarrillos hace que llegue al organismo más nicotina y alquitrán. Todo ello contribuye a la baja percepción del riesgo de fumar y a la minimización del efecto nocivo sobre la salud. ${ }^{12}$

El tabaquismo constituye un importante factor de riesgo en el cuadro de morbilidad y mortalidad en Cuba. Según las estadísticas de salud, las primeras causas de muerte en el país se encuentran relacionadas con el estilo de vida y el tabaquismo es una práctica que se señala como uno de los factores de riesgo de mayor importancia. Desde hace varias décadas, con ligeras variaciones, aparecen entre los primeros lugares en la mortalidad general enfermedades prevenibles en un alto grado, si se controlan los factores que las propician, entre ellos esta adicción. ${ }^{13}$

Cuba es el país productor del considerado mejor tabaco del mundo, por lo que su cultivo, producción y consumo están arraigados a su idiosincrasia, cultura, tradiciones y luchas sociopolíticas. Los estudios realizados han permitido conocer que el cubano se considera un pueblo de fumadores y que el tabaco constituye un importante renglón económico. ${ }^{14}$

Según la primera y segunda Encuesta Nacional de Factores de Riesgo y Actividades Preventivas de Enfermedades no Transmisibles, realizadas en 1995 y 2001 respectivamente, por el Instituto Nacional de Higiene Epidemiología y Microbiología y la Oficina Nacional de Estadísticas, en población de 17 años y más de zonas urbanas, la prevalencia nacional era en el 2001 de 31,9 \% vs. 36,8 \% en 1995 (hombres: $41,4 \%$ vs. $48,2 \%$; mujeres: $23 \%$ vs. $26,2 \%$ ).

La encuesta resalta que $63,2 \%$ no son fumadores, de ellos $10,4 \%$ son exfumadores y el resto, 52,8 \%, nunca ha fumado; también arrojó relaciones 
negativas muy importantes entre el tabaquismo y la discapacidad, la morbilidad, la mortalidad y la calidad de vida. ${ }^{15,16}$

El policlínico "Dr. Jorge Ruiz Ramírez"del municipio Playa de La Habana, según sus registros estadísticos e informes del Análisis de la Situación de Salud de 2006, presenta entre las primeras causas de muertes: tumores malignos, enfermedades cardiovasculares, cerebrovasculares y enfermedades respiratorias, las que tienen al tabaquismo como factor de riesgo, por lo que resulta significativo conocer la prevalencia de tabaquismo en la población y su percepción del riesgo de fumar. Lo anterior permitirá a la instancia sanitaria realizar propuestas para reducir la prevalencia de fumadores y el consumo del tabaco y de este modo modificar el cuadro de salud actual.

Tomando como base lo anteriormente expuesto, se consideró importante caracterizar la población de 10 años y más del área de salud del citado policlínico, describir la prevalencia y percepción del riesgo para la salud de la nocividad del tabaquismo, sobre la base de las diferentes categorías de la OMS. Se podrá así disponer de información útil para trazar estrategias considerando las particularidades de cada grupo.

\section{MÉTODOS}

Se realizó un estudio descriptivo transversal en individuos pertenecientes al área de salud del policlínico "Dr. Jorge Ruiz Ramírez", en el período comprendido entre enero y diciembre de 2007.

Se utilizó el muestreo por conglomerado bi-etápico con asignación proporcional en dos etapas, para una precisión deseada de 0,5\% que permitió garantizar una muestra aleatoria.

El universo estuvo constituido por la población de los 25 consultorios que conforman el área de salud. Se seleccionaron 13 consultorios médicos (conglomerados) y en cada uno de ellos se seleccionaron 10 familias, ambos por muestreo simple aleatorio. Como resultado del análisis realizado en el diseño muestral se definió un mínimo de 713 personas a encuestar.

Las variables estudiadas fueron las siguientes: edad, sexo, escolaridad, categoría ocupacional, clasificación del individuo con respecto al tabaquismo según categorías de la OMS, edad y causas de inicio, causas que lo mantienen, percepción e identificación de riesgos. ${ }^{17}$

La revisión documental y bibliográfica fueron los métodos iniciales. Como instrumento de recogida de información se empleó un cuestionario validado en la Encuesta Nacional de Factores de Riesgo y Enfermedades Crónicas no Trasmisibles en la población de 17 y más años, el que fue adaptado de acuerdo a los intereses del estudio y probado con anterioridad.

Las entrevistas se realizaron en el hogar, por estudiantes de medicina, previamente entrenados con el propósito de homogeneizar la toma de información.

Se tuvieron siempre presentes los principios de la ética en la investigación. Antes de aplicar la encuesta se explicó a los participantes, de forma individual y detallada, en que consistía el estudio y las ventajas que ello representaría para su comunidad, 
la voluntariedad o no para participar y la importancia de la veracidad de las respuestas. Todos expresaron su consentimiento para formar parte de la iinvestigación, en el caso de los menores de edad se les solicitó permiso a los padres o tutores para su incorporación.

Los datos se procesaron por computadoras. Se emplearon técnicas estadísticas univariadas, distribuciones de frecuencia y cálculo de porcentajes. La estimación de la prevalencia poblacional se realizó con una confiabilidad del 95\%.

\section{RESULTADOS Y DISCUSIÓN}

El cuestionario se aplicó al total de la muestra prevista para la investigación. Los individuos de menos edad tenían entre 10 y 19 años y los de mayor, más de 60 años, prevaleció el sexo femenino, el nivel escolar más frecuente fue el medio superior y predominaron los trabajadores, entre estudiantes, jubilados, amas de casa y trabajadores por cuenta propia. No hubo desempleados en la muestra.

Los sujetos incluidos en el estudio clasificados acorde a los criterios de la OMS para el tabaquismo se presentan en la tabla 1.

Tabla 1. Distribución de la población según las categorías de la..MS para el tabaquismo

\begin{tabular}{|l|c|c|}
\hline Tabaquismo & No. & $\%$ \\
\hline Fumador & 273 & 38,2 \\
\hline No fumador & 242 & 33,9 \\
\hline Fumador pasivo & 61 & 8,5 \\
\hline Ex-fumador & 56 & 7,8 \\
\hline Fumador ocasional & 49 & 6,9 \\
\hline Fumador experimental & 27 & 3,8 \\
\hline Fumador en cesación & 7 & 0,9 \\
\hline Total & 715 & 100,0 \\
\hline
\end{tabular}

La mayor proporción de sujetos fueron los fumadores activos en el momento de la encuesta, 38,2 \%, lo que demuestra una alta prevalencia de tabaquismo, que resultó inferior a la estimada para Ciudad de la Habana, 39\%, y superior a la nacional, 31,9\%, en población urbana en el 2001.3,7 Al compararla con la más reciente información mundial en que Cuba ocupa el lugar 42, solo se encuentran 15 países con prevalencias superiores a la encontrada en este territorio del municipio Playa de La Habana. ${ }^{18}$

Si se tiene en cuenta en conjunto las categorías fumador, ex-fumador, fumador ocasional, fumador experimental y fumador en cesación, se puede afirmar que el $57,6 \%$ de la población estudiada refirió fumar o haber fumado alguna vez, lo que se consideró un aspecto negativo para la salud general de la población de esta área. 
La categoría de ex-fumador representó el 7,8\%, estos resultados son discretamente inferiores a los encontrados en investigaciones anteriores en el contexto nacional ${ }^{3,7}$ y superior a los encontrado en otra investigación de la literatura internacional [UATS Parte Nacional (9 Septiembre 2003) Centro Alemán de Investigación de Cáncer, (OKFZ), Niños fumadores involuntarios son más prósperos a enfermedades, Berlín, Septiembre 7/2003, Notimec].

La proporción de fumadores resultó mayor en los grupos de edades de 40 a 49 años, $71,4 \%$ y 50 a 59 años, 63,3 \%, lo que coincide con los resultados de la literatura nacional donde la mayor prevalencia corresponde al grupo de edad 40 a 49 años. ${ }^{3,7}$

Los fumadores pasivos presentaron prevalencia alta en las edades entre 40 y 49 años y relativamente alta en los de 10 a 19 años y de 50 a 59 años. Según datos del 2006, la exposición al humo ambiental del tabaco en Cuba es alta: más de la mitad de las familias cubanas, $55 \%$ de los niños, $51 \%$ de las embarazadas y $60 \%$ de los adolescentes, están expuestos a este agente contaminante, lo que ubica a Cuba en el lugar 22 de los países de mayor prevalencia de tabaquismo pasivo en el hogar entre los 98 que enviaron datos a la Organización Mundial de la Salud, y el tercer lugar entre los 26 países de América que informaron este dato, superado solo por Argentina, $70 \%$ y Uruguay $63 \% .{ }^{19}$

Los fumadores que tratan de dejar la adicción, categoría "en cesación", presentaron prevalencias muy bajas o nulas, siendo reflejo de ello la relativamente baja prevalencia de exfumadores en la muestra, sobre todo en los menores de 50 años.

La baja prevalencia de la categoría exfumador y la alta prevalencia de fumadores (tabla 1), demuestra el carácter adictivo del tabaquismo y lo difícil que resulta la deshabituación, comportamiento que es similar al de cualquier drogadicción. ${ }^{3,7}$

Se observaron diferencias en las categorías del tabaquismo de la OMS y el sexo de los encuestados, según se aprecia en la tabla 2.

Tabla 2. Distribución de la población según categorias de tabaquismo

de la OMS y sexo

\begin{tabular}{|c|c|c|c|c|}
\hline \multirow{2}{*}{$\begin{array}{l}\text { Categorias de } \\
\text { tabaquismo de la OMS }\end{array}$} & \multicolumn{2}{|c|}{ Masculino } & \multicolumn{2}{|c|}{ Femenino } \\
\hline & No. & $\%$ & No. & $\%$ \\
\hline Fumador & 160 & 46,7 & 113 & 30,4 \\
\hline No fumador & 99 & 28,9 & 143 & 38,4 \\
\hline Fumador pasivo & 17 & 4,9 & 44 & 11,8 \\
\hline Ex-fumador & 19 & 5,6 & 37 & 9,9 \\
\hline Fumador ocasional & 24 & 6,9 & 25 & 6,7 \\
\hline Fumador experimental & 21 & 6,1 & 6 & 1,6 \\
\hline Fumador en cesación & 3 & 0,9 & 4 & 1,1 \\
\hline Total & 343 & 100,0 & 372 & 100,0 \\
\hline
\end{tabular}


Las proporciones de fumadores y fumadores experimentales fueron mayores en el sexo masculino, mientras que las proporciones de no fumadores, fumador pasivo y exfumador, resultaron mayores en el sexo femenino. Las categorías fumadores en cesación y ocasionales en ambos sexos presentaron proporciones semejantes. Es apreciable que el sexo masculino es el de mayor prevalencia de tabaquismo y a pesar de la tendencia a aumentar el consumo de tabaco en el sexo femenino todavía existen diferencias, lo que apunta hacia la importancia de definir las estrategias preventivas con enfoque de género.

Las categorías del tabaquismo de la OMS y el nivel escolar de los encuestados presentaron diferencias. Las proporciones de fumadores, fumadores ocasionales, experimentales y pasivos resultaron mayores en los niveles escolares de secundaria, medio superior y universitario, mientras que la proporción de no fumadores lo resultó en el nivel escolar de primaria. La categoría fumador en cesación presentó proporciones muy pequeñas y semejantes. Los estudios revisados no permiten establecer comparaciones con el nivel escolar y las categorías de tabaquismo por solo referirse a la prevalencia poblacional.

Si se compara la categoría de fumador y la escolaridad, se puede afirmar que aunque el nivel que predominó en la población estudiada es el medio superior, en el grupo de fumadores se observó una prevalencia mayor en el nivel universitario, $64,8 \%$. Resultados como este se obtuvieron en la encuesta de factores de riesgo en el año 1995, no así en la aplicada en el año 2001 donde prevaleció el nivel de primaria seguido del de secundaria básica. ${ }^{3,7}$

Se observaron diferencias en las categorías del tabaquismo de la OMS y la clase ocupacional de los encuestados. Las proporciones de fumadores y fumadores ocasionales fueron mayores en los estudiantes, trabajadores y jubilados, mientras que las proporciones de ex-fumador, fumador pasivo y experimental lo fueron en las amas de casa. Los no fumadores tenían mayor proporción que los fumadores en las amas de casa pero menor en los trabajadores y jubilados. La categoría fumador en cesación presentó proporciones muy pequeñas semejantes.

Los valores de prevalencia poblacional estimados corroboran el mayor riesgo de tabaquismo en estudiantes, trabajadores y jubilados (Hernández J, Lorena Guevara C, García M, Jorge E. Hábito de fumar en los estudiantes de primer semestre de la Facultad de Salud: características y percepciones. Universidad del Valle; 2003). También la prevalencia del fumador pasivo fue mayor en amas de casa y trabajadores. Al analizar estos resultados según los valores de prevalencia poblacional, la categoría ocupacional trabajadores es la de mayor riesgo de tabaquismo y de fumador pasivo, lo cual demuestra que se mantiene la aceptación social y que en los centros de trabajo no se respeta totalmente lo que plantean los diferentes documentos legislativos sobre la prohibición de fumar en lugares públicos. ${ }^{20,21}$

Es importante conocer la edad de inicio del tabaquismo por el síndrome de dependencia que puede crear; si se empieza a fumar a edades tempranas, es más probable que se continué fumando en la adultéz, es menos probable que se deje el hábito y el tiempo en que será consumidor será más largo, con lo cual aumentan los riesgos de desarrollar enfermedades crónicas.

Los resultados entre las categorías de la OMS para el tabaquismo y la edad de inicio, se muestran en la tabla 3. 
Tabla 3. Distribución de la población según categorías de tabaquismo de la. OMS

y grupos de edad de inicio del tabaquismo

\begin{tabular}{|c|c|c|c|c|c|c|c|c|c|c|c|c|}
\hline \multirow{3}{*}{$\begin{array}{l}\text { Grupo de } \\
\text { edad e } \\
\text { inicio del } \\
\text { tabaquismo }\end{array}$} & \multicolumn{10}{|c|}{ Categorías de tabaquismo de la OMS } & & \\
\hline & \multicolumn{2}{|c|}{ Fumador } & \multicolumn{2}{|c|}{$\begin{array}{l}\text { Ex- } \\
\text { fumador }\end{array}$} & \multicolumn{2}{|c|}{$\begin{array}{l}\text { Fumador } \\
\text { ocasional }\end{array}$} & \multicolumn{2}{|c|}{$\begin{array}{l}\text { Fumador } \\
\text { experimental }\end{array}$} & \multicolumn{2}{|c|}{$\begin{array}{l}\text { Fumador } \\
\text { en } \\
\text { cesación }\end{array}$} & \multicolumn{2}{|c|}{ Total } \\
\hline & No. & $\%$ & No. & $\%$ & No. & $\%$ & No. & $\%$ & No. & $\%$ & No. & $\%$ \\
\hline 10 a 15 & 38 & 50,6 & 11 & 14,7 & 17 & 22,7 & 9 & 12,0 & - & - & 75 & 18,2 \\
\hline 16 a 20 & 148 & 67,6 & 27 & 12,3 & 25 & 11,4 & 12 & 5,5 & 7 & 3,2 & 219 & 53,2 \\
\hline 21 a 25 & 79 & 75,2 & 15 & 14,3 & 5 & 4,8 & 6 & 5,7 & - & - & 105 & 25,5 \\
\hline 26 a 30 & 8 & 61,5 & 3 & 23,1 & 2 & 15,4 & - & - & - & - & 13 & 3,1 \\
\hline Total & 273 & 100 & 56 & 100 & 49 & 100 & 27 & 100 & 7 & 100 & 412 & 100 \\
\hline
\end{tabular}

La mayor proporción de fumadores resultó iniciarse en la adicción entre los 16 y 20 años, $53,2 \%$ y el $18,2 \%$ lo hizo antes de los 16 años, lo que permitió conocer que $71,4 \%$ de los fumadores se iniciaron antes de los 20 años de edad. Similares resultados se han obtenido en la literatura nacional. ${ }^{22}$ Según otras investigaciones de la literatura internacional se ha encontrado la edad de inicio antes de los 15 años. ${ }^{23}$

La causa de inicio más frecuente mencionada resultó ser "mis amigos me estimularon", 33,5 \%, le siguieron en orden descendente, "por embullo", 24,5\%, "mis padres fumaban", 17,0\%, "me resultaba interesante", 10,0\%, "para mostrar madurez", 7,5 \%, "por stress"3,9 \% y "para mantener la vigilia", 3,6 \%. En otros estudios se encuentran causas de inicio similares aunque con variaciones en cuanto al ordenamiento. ${ }^{24}$

Al analizar las causas de inicio en el hábito por las categorías de la OMS se apreciaron diferencias tales como:

- Ios fumadores, fumadores ocasionales, fumadores experimentales y fumadores en cesación, señalaron en primer orden "mis amigos me estimularon", pero los exfumadores en segundo orden y su principal causa fue "por embullo",

- los fumadores experimentales tuvieron una proporción mayor que las demás categorías en las causas "mis padres fumaban" y "me resultaba interesante",

- la causa "para mantener la vigilia"resultó mayor en fumadores ocasionales que en las demás categorías,

- la causa "para mostrar madurez", fue señalada en mayor proporción por los fumadores activos.

No se han encontrado estudios anteriores que permitan realizar comparaciones para cada categoría, no obstante, constituyen una información de gran valor para diseñar estrategias de prevención y cesación a nivel local, 
La causa más frecuentemente mencionada para el mantenimiento de la adicción fue "por placer" por más de la mitad de los incluidos en este análisis, 51,4\%. Le siguieron muy distantes las otras causas señaladas, siendo en orden descendente "por embullo", 12,1\%, "por stress", 11,2 \%, "falta de voluntad""para mantener vigilia", 8,1\%. La causa menos mencionada fue "todos en casa lo hacen", 2,4\%.

Como se puede apreciar, "por placer", fue la causa por la que mantiene la adicción la mayor parte de los fumadores. En relación con el fumador activo, hay investigadores que también han encontrado "el placer" como la principal causa de la adición; si a esto se le suma el efecto a largo plazo de la nocividad a la salud y el carácter adictivo, el cambio que se requiere en el orden cognoscitivo, de actitud y conductual, resulta muy complejo. ${ }^{25}$

Al analizar las causas de mantener la adicción por las categorías de la OMS se apreciaron las diferencias siguientes:

- los fumadores, exfumadores, fumadores en cesación y experimentales, señalaron "el placer" en primer orden, pero los fumadores ocasionales en segundo orden y su principal causa fue "el embullo",

- los fumadores en cesación presentaron una proporción mayor que las demás categorías en la causa "por embullo",

- la causa del "stress"resultó mayor en fumadores ocasionales y en exfumadores que en las demás categorías,

- la causa para "mantener la vigilia"fue mayor en los exfumadores que en el resto,

- la "falta de voluntad"fue señalada solamente por los fumadores actuales activos.

Estos resultados no pueden ser comparados con los encontrados en la bibliografía consultada debido a que no se abordan todas las categorías de tabaquismo según la OMS relacionados con este aspecto. Es de destacar la importancia que tiene que los fumadores activos señalen "la falta de voluntad" para cesar la adicción, pues precisamente este debe ser el punto central de la estrategia encaminada a lograr que dejen de fumar y que se les ofrezca la ayuda requerida para la deshabituación, a partir de la necesidad que tienen de terapias y grupos de apoyo, así como de medidas externas, tales como la prohibición de fumar en lugares públicos, a lo que se le puede añadir la importancia de que en el hogar se vele por la no contaminación del aire para proteger a los no fumadores, fundamentalmente a los niños.

Es conocido que aunque las personas tengan conocimientos sobre la nocividad de determinados comportamientos y consumo de productos, el hecho de que los efectos no se produzcan a corto plazo hace que los riesgos se minimicen y se despersonalicen. De la situación anterior no se encuentran exentos ni los profesionales sanitarios, como ya se ha planteado. Es por eso que resulta tan interesante disponer de información que permita diagnosticar, tanto los conocimientos sobre los daños, como la forma en que estos son percibidos por la población sobre la cual se quiere intervenir.

La tabla 4 muestra los sujetos encuestados y la percepción del riesgo del tabaquismo, según las categorías de la OMS. 
Tabla 4. Distribución de la población según percepción del riesgo del tabaquismo por categorías de la..OMS

\begin{tabular}{|c|c|c|c|c|c|c|c|c|}
\hline \multirow{3}{*}{$\begin{array}{l}\text { Categorías de } \\
\text { tabaquismo de la } \\
\text { OMS }\end{array}$} & \multicolumn{8}{|c|}{ Percepción del riesgo del tabaquismo } \\
\hline & \multicolumn{2}{|c|}{ Hay riesgo } & \multicolumn{2}{|c|}{$\begin{array}{l}\text { No hay } \\
\text { riesgo }\end{array}$} & \multicolumn{2}{|c|}{ No sabe } & \multicolumn{2}{|c|}{ Total } \\
\hline & No. & $\%$ & No. & $\%$ & No. & $\%$ & No. & $\%$ \\
\hline Fumador & 256 & 93,8 & 4 & 1,5 & 13 & 4,7 & 273 & 100,0 \\
\hline No fumador & 232 & 95,9 & 6 & 2,5 & 4 & 1,6 & 242 & 100,0 \\
\hline Fumador pasivo & 57 & 93,4 & 2 & 3,3 & 2 & 3,3 & 61 & 100,0 \\
\hline Ex-fumador & 55 & 98,2 & 1 & 1,8 & - & - & 56 & 100,0 \\
\hline Fumador ocasional & 42 & 85,7 & 4 & 8,2 & 3 & 6,1 & 49 & 100,0 \\
\hline $\begin{array}{l}\text { Fumador } \\
\text { experimental }\end{array}$ & 24 & 88,9 & 2 & 7,4 & 1 & 3,7 & 27 & 100,0 \\
\hline Fumador en cesación & 6 & 85,7 & - & - & 1 & 14,3 & 7 & 100,0 \\
\hline Total & 672 & 93,9 & 19 & 2,7 & 24 & 3,4 & 715 & 100,0 \\
\hline
\end{tabular}

Se aprecia que la inmensa mayoría de los encuestados perciben que el tabaquismo es un riesgo, 93,9\%, una exigua minoría, $2,7 \%$ refiere que no es un riesgo y $3,4 \%$ no sabe al respecto. Otros estudios de la literatura nacional e internacional han demostrado que la población conoce de la nocividad del tabaco, pero todavía no tiene una concepción clara de la percepción del riesgo. ${ }^{25,26}$

En el presente estudio, el conocimiento de las enfermedades que el encuestado considera que se producen como consecuencia del tabaquismo se refleja en la tabla $\underline{5}$.

Tabla 5. Enfermedades que el encuestado considera se producen como consecuencia del tabaquismo

\begin{tabular}{|l|l|l|}
\hline Enfermedades & No. & $\%$ \\
\hline Cáncer del pulmón & 658 & 97,9 \\
\hline Otros cánceres & 71 & 10,5 \\
\hline Enfisema pulmonar & 22 & 3,2 \\
\hline Bronquitis crónica & 13 & 1,9 \\
\hline
\end{tabular}

Como se aprecia, la enfermedad más conocida fue el cáncer de pulmón. Otras enfermedades señaladas en el modelo de encuesta no fueron consideradas como riesgo. Estos resultados denotan un real desconocimiento de los riesgos que entraña el tabaquismo en la población estudiada, pues muchas enfermedades no son referidas y sin embargo está demostrado su relación con la morbilidad y mortalidad. Otros autores han encontrado resultados similares. ${ }^{27,28}$ 
El estudio realizado permite concluir, que el tabaquismo constituye un importante factor de riesgo en la morbilidad y mortalidad en el área de salud del policlínico "Dr. Jorge Ruiz Ramírez", dada la elevada prevalencia encontrada, los grupos de edad de mayor frecuencia y la temprana edad de inicio, así como el hecho de que la población desconoce las enfermedades relacionadas con el tabaquismo, aunque declaran que constituye un riesgo para la salud,

Caracterizar el tabaquismo en la población, de acuerdo a la clasificación de la OMS, constituye una información de gran valor para trazar estrategias de prevención y control en las áreas de salud que permitan intervenir, a nivel local, con un enfoque multidisciplinario e intersectorial y diseñar acciones para cada grupo poblacional.

\section{REFERENCIAS BIBLIOGRÁFICAS}

1. Ordúñez PO, Cooper RS, Espinosa AD, Iraola Ferrer MD. Enfermedades cardiovasculares en Cuba: determinantes para una epidemia y desafíos para la prevención y control. Rev Cubana Salud Pública. 2005;31(4):58-9.

2. Rial Blanco N. El control para la prevención y el control del tabaquismo del INACV. Rev Cubana Angiología y Cirugía Vascular. 2000;1:32.

3. Instituto Nacional de Investigación sobre Tabaco. Boletín para el control del tabaco. Cuernavaca, México. Septiembre 2004,p.7.

4. López García-Aranda V, García Rubira J. Tabaco y enfermedad coronaria [sitio en Internet]. [citado 2008]. Disponible en:

http://www.crf.medynet.com/contenido/2000/6/408-416.pdf

5. Organización Panamericana de la salud. Tres mediciones fundamentales para revitalizar el control del tabaquismo en Las Américas. Washington, D.C.: OPS;2002. [Publicación científica Vol, 11, No. 2].

6. Organización Panamericana de la Salud. Tabaquismo: un desastre para la salud pública. Washington, D.C.: OPS;1997.

7. Instituto Nacional de Salud Pública de México (INSP). Perspectivas de control del tabaquismo en América Latina y el Caribe [CD ROM]. México: INSP;2004.

[Información seleccionada para el curso de verano. 23-27 Agosto de 2004].

8. Valdés Salgado R, Lazcano Ponce E, Hernández Ávila M. Primer informe sobre el combate al tabaquismo. México ante el Convenio Marco para el Control del Tabaco. México, Cuernavaca: Instituto Nacional de Salud Pública;2005.

9. Suárez Lugo N. Tabaco o salud: una decisión social. Revista Horizontes Sanitarios. UJAT. 2004;3(2). [Tabasco, México].

10. Suárez Lugo N, Caraballoso Hernández M, Echevarria Negrín S. El tabaquismo en los profesionales de la salud en el municipio Habana Vieja. Rev Cubana Salud Pública. 2008;34(4).

11. Warren Ch, Riley S. Tobacco use by youth: a surveillance report from the global youth tobacco survey project. Bull WHO. 2000;78(7):20. 
12. Lezcano E. La epidemia de tabaquismo. Epidemiología, factores de riesgo y medidas de prevención. Salud Pública Méx. 2002;44 (supl. 1):5-18.

13. Suárez Lugo N. Enfoque Social de la Mercadotecnia Sanitaria. La Habana: Editorial Ciencias Médicas;2007.

14. Dirección Nacional de Registros y Estadísticas de Salud. Anuario Estadístico de Salud 2006. La Habana: MINSAP;2007.

15. Instituto Nacional de Higiene y Epidemiología. Encuesta Nacional de Factores de Riesgo. La Habana: INHEM;2002.

16. Instituto Nacional de Higiene y Epidemiología. Encuesta nacional de factores de riesgo. La Habana: INHEM;1996.

17. Campos Pupo Elizabeth. Prevalencia y percepción del riesgo del tabaquismo. Población 10 años y más. Área de Salud del policlínico "Dr. Jorge Ruiz Ramírez", Ciudad de la Habana, 2007 [tesis]. La Habana: Escuela Nacional de Salud Pública;2009.

18. Mackay J, Eriksen M, Shafey O, Ross H. The tobacco atlas. 3th ed. Atlanta, Georgia USA: American Cancer Society, World Lung Foundation;2009.

19. Mackay J, Eriksen M, Shafe O. The tobacco atlas. 2nd ed. Reino Unido, Myraid: American Cancer Society;2006.

20. Varona Pérez P. Tabaquismo y sus características en trabajadores de salud en el municipio Centro Habana. Rev Cubana Med Gen Integr. 2000;16(3):22-6.

21. Suárez Lugo N, Caraballoso Hernández M, Rodríguez Cuelez D. Legislación para el control del tabaquismo en Cuba. Rev Cubana Salud Pública. 2008;34(3).

22. Lancés Cotilla L. Segunda encuesta Mundial sobre tabaquismo en jóvenes, Cuba 2004. La Habana: MINSAP;2005. [Informe de investigación. Ejecución: INHEM, ENSAP].

23. Organización Mundial de la Salud. Encuesta mundial sobre el tabaco en jóvenes [sitio en Internet]. [citado 2008]. Disponible en:

http://www.radiomundial.com.ve/yvke/noticia.php?32415

24. Tabaquismo en profesionales de la salud del Instituto Mexicano del Seguro Social. Morelos: Instituto;2008.

25. US Department of Health and Social Security. Women and smoking. A report of the Surgeon General, Atlanta, G. A.: Office Smoking and Health;2001. [CDC, 2].

26. Suárez Lugo N. El consumo de productos manufacturados del tabaco en Cuba. Rev Cubana Salud Pública. 2006; 32(2):102-10.

27. Los médicos españoles admiten que se siente impotentes frente al tabaquismo [sitio en Internet]. [citado 16 de septiembre de 2007]. Disponible en: http://www.larioja.com/prensa/20060905/sociedad/medicos-espanoles-dicensienten 20060905.html 
28. Espinosa EO, Pardo E, Gózales Díaz M. El consenso para dejar de fumar: por qué, cómo y cuándo, debemos de darlo a nuestros pacientes. Aten Primaria. $2005 ; 36(1): 46-9$.

Recibido: 13 de abril de 2009.

Aprobado: 2 de mayo de 2009.

Nery Suárez Lugo. Escuela Nacional de Salud Pública. Calle I No.202 e/ 11 y Línea. El Vedado10400. La Habana, Cuba.

E-mail: nerysl@infomed.sld.cu 